\title{
Dehalogenimonas lykanthroporepellens gen. nov., sp. nov., a reductively dehalogenating bacterium isolated from chlorinated solvent-contaminated groundwater
}

Correspondence

William M. Moe

moemwil@lsu.edu

\author{
William M. Moe, ${ }^{1}$ Jun Yan, ${ }^{1}$ M. Fernanda Nobre, ${ }^{2}$ Milton S. da Costa ${ }^{3}$ \\ and Fred A. Rainey ${ }^{4}$ \\ ${ }^{1}$ Department of Civil and Environmental Engineering, Louisiana State University, Baton Rouge, \\ LA 70803, USA \\ ${ }^{2}$ Departamento de Zoologia and Centro de Neurociências, Universidade de Coimbra, 3004-517 \\ Coimbra, Portugal \\ ${ }^{3}$ Departamento de Bioquímica and Centro de Neurociências, Universidade de Coimbra, 3001-401 \\ Coimbra, Portugal \\ ${ }^{4}$ Department of Biological Sciences, Louisiana State University, Baton Rouge, LA 70803, USA
}

Two recently reported bacterial strains that are able to reductively dehalogenate polychlorinated aliphatic alkanes, including 1,2,3-trichloropropane, 1,2-dichloropropane, 1,1,2,2-

tetrachloroethane, 1,1,2-trichloroethane and 1,2-dichloroethane, were further characterized to clarify their taxonomic position. The two strains, designated BL-DC-8 and BL-DC- $9^{\top}$, were mesophilic, non-spore-forming, non-motile, Gram-negative staining and strictly anaerobic. Cells were irregular cocci, 0.3-0.6 $\mu \mathrm{m}$ in diameter. The two strains were resistant to ampicillin and vancomycin. Hydrogen was utilized as an electron donor. The genomic DNA G+C content of strains BL-DC-8 and BL-DC-9 ${ }^{\top}$ was 54.0 and 53.8 mol\%, respectively. The major cellular fatty acids were $\mathrm{C}_{18: 1} \omega 9 c, \mathrm{C}_{16: 1} \omega 9 c, \mathrm{C}_{16: 0}$ and $\mathrm{C}_{14: 0}$. Phylogenetic analyses based on 16S rRNA gene sequences indicated that the strains cluster within the phylum Chloroflexi, but are related only distantly to all recognized taxa in the phylum. Morphological, physiological and chemotaxonomic traits as well as phylogenetic analysis support the conclusion that these two strains represent a novel species of a new genus in the phylum Chloroflexi, for which the name Dehalogenimonas lykanthroporepellens gen. nov., sp. nov. is proposed. The type strain of Dehalogenimonas lykanthroporepellens is BL-DC-9 ${ }^{\top}$ (=ATCC BAA-1523 ${ }^{\top}=\mathrm{JCM} 15061^{\top}$ ).
The phylum Chloroflexi is a deep branching lineage within the domain Bacteria. The phylum contains at least six major lineages, five of which, at the time of writing, have cultured representatives. These lineages include the classes Anaerolinea (Yamada et al., 2006), Caldilinea (Yamada et al., 2006), 'Chloroflexi' (Castenholz, 2001) and Thermomicrobia (Hugenholtz \& Stackebrandt, 2004). Also included in the phylum Chloroflexi is a lineage for which the class 'Dehalococcoidetes' was informally proposed by Hugenholtz \& Stackebrandt (2004) to accommodate the

The GenBank/EMBL/DDBJ accession numbers for the 16S rRNA gene sequences of strains BL-DC-9 ${ }^{\top}$ and BL-DC-8 are EU679419 and EU679418, respectively.

Scanning electron and transmission electron micrographs of cells of strains BL-DC-9 ${ }^{\top}$ and BL-DC-8 and phenotypic characteristics of type species of genera in the phylum Chloroflexi are available with the online version of this paper. tetrachloroethene-respiring coccus 'Dehalococcoides ethenogenes' (Maymó-Gatell et al., 1997).

Two novel anaerobic bacterial strains that cluster within the phylum Chloroflexi, designated BL-DC-8 and BL-DC$9^{\mathrm{T}}$, were recently demonstrated to be able to reductively dehalogenate a variety of polychlorinated alkanes (Yan et al., 2009). These represent the first strains isolated in pure culture to be able anaerobically to reductively dehalogenate 1,2,3-trichloropropane. For each of these strains, 1,2,3-trichloropropane was transformed to the intermediate allyl chloride (3-chloro-1-propene). Allyl chloride is unstable and underwent abiotic hydrolysis to form allyl alcohol. Allyl chloride also underwent abiotic reactions with sulfide and cysteine, reducing agents which were present in the growth medium, to form various other compounds, including diallyl sulfide, diallyl disulfide, allyl mercaptan and allyl methyl sulfide. These final products 
are compounds that contribute to the recognizable odour associated with garlic (Laakso et al., 1989; Lawson, 1996). The two strains were isolated from groundwater collected from a waste recovery well at the PetroProcessors of Louisiana, Inc. Superfund Site, near Baton Rouge, LA, USA, an area heavily contaminated with chlorinated solvents (Bowman et al., 2006). Data from the present study show that strains BL-DC- $9^{\mathrm{T}}$ and BL-DC-8 represent a single novel species of a new genus in the phylum Chloroflexi.

The genomic DNA G + C content of strains BL-DC-8 and BL-DC- $9^{\mathrm{T}}$ was determined by HPLC as described by Mesbah et al. (1989) following DNA isolation by using an UltraClean microbial DNA isolation kit (MoBio Laboratories Inc.).

Cells for analysis of cellular fatty acids were grown in anaerobic basal medium prepared as described by Yan et al. (2009) except that the sodium sulfide solution was replaced with titanium citrate solution (Zehnder \& Wuhrmann, 1976) as a reducing agent at a final concentration of $1.0 \mathrm{mM} \mathrm{Ti}(\mathrm{III})$ and $2.0 \mathrm{mM}$ citrate. The medium was supplemented with $5 \mathrm{mM}$ sodium acetate and $0.5 \mathrm{mM}$ 1,1,2-trichloroethane, and provided with hydrogen gas $(10 \%, \mathrm{v} / \mathrm{v})$ in the headspace. Cells were harvested via centrifugation $(10000 \mathrm{~g})$ following static incubation in the dark at $30{ }^{\circ} \mathrm{C}$ for 2 weeks. Cellular fatty acids were extracted, saponified and methylated according to the protocol of the Sherlock Microbial Identification System (MIDI). The fatty acids were analysed by using a gas chromatograph equipped with the Microbial Identification software package (Sasser, 1990).

Scanning electron microscopy was performed as described by Yan et al. (2009). For transmission electron microscopy, cells were collected and fixed in the same manner as for scanning electron microscopy. Preparations were rinsed with $0.02 \mathrm{M}$ glycine in $0.1 \mathrm{M}$ cacodylate buffer, post-fixed in $2 \%$ osmium tetroxide and stained with $0.5 \%$ uranyl acetate. Preparations were dehydrated with a graded ethanol series. The membrane was infiltrated with $1: 1$ ethanol/LR white embedding resin and infiltrated and embedded with $100 \%$ resin. Samples were sectioned with a DuPont MT5000 ultramicrotome and stained with Reynolds' lead citrate. Cross-sections were imaged with a JEOL 100CX transmission electron microscope.

Genomic DNA extraction, PCR amplification and $16 \mathrm{~S}$ rRNA gene sequencing were performed as described by Yan et al. (2009). Phylogenetic analyses were performed by using the ARB program (Ludwig et al., 2004). The neighbour-joining algorithm was used to build the phylogenetic tree, with Jukes-Cantor correction (Jukes \& Cantor, 1969) followed by bootstrap analysis with PHYLIP 3.62 (Felsenstein, 1985) based on 1000 replications.

The genomic DNA G + C contents of strains BL-DC- 8 and BL-DC- $9^{\mathrm{T}}$ were 54.0 and $53.8 \mathrm{~mol} \%$, respectively. The major cellular fatty acids included $\mathrm{C}_{18: 1} \omega 9 c, \mathrm{C}_{16: 1} \omega 9 c$,
Table 1. Cellular fatty acid profiles of strains BL-DC-8 and BL-DC-9 ${ }^{\top}$

Values are percentages of the total fatty acids. -, Not detected.

\begin{tabular}{|lcc|}
\hline Fatty acid & BL-DC-8 & BL-DC-9 $^{\text {T }}$ \\
\hline $\mathrm{C}_{9: 0}$ & 0.4 & 0.3 \\
$\mathrm{C}_{10: 0}$ & 0.5 & 0.4 \\
$\mathrm{C}_{12: 0}$ & 1.8 & 1.9 \\
Anteiso-C $14: 0$ & 0.6 & 0.5 \\
$\mathrm{C}_{14: 0}$ & 11.8 & 13.3 \\
$\mathrm{C}_{15: 0}$ & - & 0.4 \\
$\mathrm{C}_{16: 1} \omega 9 c$ & 23.0 & 23.2 \\
Summed feature $3 *$ & 2.7 & 2.1 \\
$\mathrm{C}_{16: 0}$ & 17.1 & 20.8 \\
Summed feature $5^{*}$ & 7.9 & 6.5 \\
$\mathrm{C}_{18: 1} \omega 9 c$ & 21.8 & 23.4 \\
Summed feature $8^{*}$ & 4.7 & - \\
$\mathrm{C}_{18: 0}$ & 7.1 & 7.1 \\
$\mathrm{C}_{19: 0}$ cyclo $\omega 8 c$ & 0.7 & - \\
$\mathrm{C}_{20: 1} \omega 9 c$ & - & 0.6 \\
\hline
\end{tabular}

* Summed features are groups of two or three fatty acids that cannot be separated by GLC using the MIDI system. Summed feature 3 contains one or more of $\mathrm{C}_{16: 1} \omega 7 c, \mathrm{C}_{16: 1} \omega 6 c$ and iso- $\mathrm{C}_{15: 0} 3-\mathrm{OH}$; summed feature 5 contains $\mathrm{C}_{18: 2} \omega 6,9 c$ and/or anteiso- $\mathrm{C}_{18: 0}$; summed feature 8 contains $\mathrm{C}_{18: 1} \omega 7 c$ and/or $\mathrm{C}_{18: 1} \omega 6 c$.

$\mathrm{C}_{16: 0}$ and $\mathrm{C}_{14: 0}$ (Table 1). Apart from the presence of summed feature 8 (comprising $\mathrm{C}_{18: 1} \omega 7 c$ and/or $\mathrm{C}_{18: 1} \omega 6 c$ ) as $4.7 \%$ of the total in strain BL-DC- 8 and its absence from strain BL-DC- $9^{\mathrm{T}}$, there were only minor differences in the cellular fatty acid contents of the two strains. Cells of the two strains were non-spore-forming, irregular cocci, 0.3$0.6 \mu \mathrm{m}$ in diameter (see Supplementary Fig. S1 in IJSEM Online). Additional phenotypic characteristics were described previously (Yan et al., 2009) and are included in the species description. The two strains shared nearly identical phenotypic properties, exhibiting identical substrate utilization profiles and differing only slightly in temperature range for growth $\left(20-34{ }^{\circ} \mathrm{C}\right.$ for strain BL-DC8 and $20-37{ }^{\circ} \mathrm{C}$ for strain BL-DC- $\left.9^{\mathrm{T}}\right)$. The two strains exhibited reductive dechlorination of 1,2-dichloropropane, a growth-related process, in the $\mathrm{pH}$ range $6.0-8.0$ but not at $\mathrm{pH} \leqslant 5.5$ or $\geqslant 8.5$ during a 2 -month incubation period (Yan et al., 2009). As the groundwater at the location from which the strains were isolated had a $\mathrm{pH}$ of 5.1 (Bowman et al., 2006), the habitat of these strains probably extends to $\mathrm{pH}$ levels somewhat lower than determined in the laboratory test conditions employed by Yan et al. (2009).

Phylogenetically, strains BL-DC-8 and BL-DC- $9^{\mathrm{T}}$ were closely related, sharing $99.93 \% 16 \mathrm{~S}$ rRNA gene sequence similarity ( $1 \mathrm{nt}$ difference). In the neighbour-joining tree, the novel strains were placed distantly from recognized genera within a deep branch of the Chloroflexi (Fig. 1). Their closest cultured relatives were 'Dehalococcoides' 




Fig. 1. Neighbour-joining dendrogram of the phylum Chloroflexi (Hugenholtz \& Stackebrandt, 2004) based on 16S rRNA gene sequences showing the phylogenetic relationship of strains BL-DC- $9^{\top}$ and BL-DC-8 and related environmental clones and cultured isolates without validly published names to recognized taxa. Bootstrap values expressed as percentages of 1000 resamplings are shown at branch points. Bar, 5 substitutions per 100 nucleotide positions. PCB, Polychlorinated biphenyl; TCE, trichloroethene; TeCA, 1,1,2,2-trichloroethane; VC, vinyl chloride.

strains, but with only $90 \% 16 \mathrm{~S}$ rRNA gene sequence similarity. The clustering of strains BL-DC-8 and BL-DC$9^{\mathrm{T}}$ with the 'Dehalococcoides' was supported by a bootstrap value of $94 \%$.

Maymó-Gatell et al. (1997) informally proposed the name 'Dehalococcoides ethenogenes' for a bacterial strain (strain 195) isolated from an enrichment culture derived from anaerobically digested wastewater sludge. This bacterium was the first strain isolated in pure culture that was shown to reductively dehalogenate tetrachloroethene to ethene. Additional strains with close phylogenetic relatedness to 'Dehalococcoides ethenogenes' 195 (>99\% 16S rRNA gene sequence similarity) have been reported subsequently in the literature as 'Dehalococcoides' sp. (He et al., 2003, 2005; Kube et al., 2005; Sung et al., 2006). There has been intense interest in this group of bacteria because of their ability to reductively dehalogenate a variety of chlorinated compounds that are widespread pollutants of environmental importance (Hendrickson et al., 2002; Bowman et al., 2006). At the time of writing, however, the genus 'Dehalococcoides' has not been formally described. The strain designated in the literature as 'Dehalococcoides ethenogenes' 195 (Maymó-Gatell et al., 1997) and the related 'Dehalococcoides' strains BAV1, CBDB1, FL2 and GT (Adrian et al., 2000; He et al., 2003, 2005; Sung et al., 2006) have not been deposited in public culture collections as required for valid publication under the Bacteriological Code (Lapage et al., 1992), and no formal taxonomic descriptions for these exist. The above genus and species names, however, are widely used in the literature to the extent that 'Dehalococcoides' has been carried to the class level with the informal proposal of Hugenholtz \& Stackebrandt (2004) of the class 'Dehalococcoidetes'. In order to move the taxonomy of this lineage forward and to establish an anchor point for future higher-level taxa, we propose the genus Dehalogenimonas gen. nov. to accommodate strains BL-DC- 8 and BL-DC- $9^{\mathrm{T}}$, this genus being 
related most closely to but distinct from the 'Dehalococcoides' cluster.

Although strains BL-DC-8 and BL-DC-9 $9^{\mathrm{T}}$ share several common phenotypic features with the 'Dehalococcoides' strains reported to date, including strictly anaerobic respiration, requirement for $\mathrm{H}_{2}$ as an electron donor, apparently exclusive use of halogenated compounds as electron acceptors, coupling of cell growth with reductive dehalogenation, Gram-negative staining, small irregular cell morphology and resistance to the antibiotics ampicillin and vancomycin (Adrian et al., 2000; He et al., 2003, 2005; Sung et al., 2006; Yan et al., 2009), they can be differentiated from members of the 'Dehalococcoides' cluster based on genotypic, chemotaxonomic and phenotypic properties. Based on $16 \mathrm{~S}$ rRNA gene sequence comparisons, strains BL-DC-8 and BLDC $-9^{\mathrm{T}}$ branch with but represent a distinct lineage at the $90 \%$ similarity level from 'Dehalococcoides' strains (Fig. 1). Such a low level of $16 \mathrm{~S}$ rRNA gene sequence similarity is indicative of a taxon distinct to at least the genus level. The genomic DNA G $+C$ contents of strains BL-DC-8 and BLDC- $9^{\mathrm{T}}$ (54.0 and $53.8 \mathrm{~mol} \%$, respectively) are appreciably higher than those for 'Dehalococcoides ethenogenes' 195 (48.9 mol\%; Seshadri et al., 2005), 'Dehalococcoides' sp. CBDB1 (47.0 mol\%; Kube et al., 2005) and 'Dehalococcoides' sp. BAV1 (47.1 mol\%; http://genome.ornl.gov/microbial/ deha_bav1/).

The cellular fatty acids determined for strains BL-DC- 8 and BL-DC-9 ${ }^{\mathrm{T}}$ (Table 1) differ from those reported for 'Dehalococcoides' strains BAV1 and FL2, the only 'Dehalococcoides' strains characterized to date in this regard. Strains BL-DC-8 and BL-DC- $9^{\mathrm{T}}$ contained large proportions of $\mathrm{C}_{16: 1} \omega 9 \mathrm{c}$ (23.0 and $23.2 \%$, respectively), significant concentrations of summed feature 5 (one or more of $\mathrm{C}_{18: 2} \omega 6 c, \mathrm{C}_{18: 2} \omega 9 c$ and anteiso- $\mathrm{C}_{18: 0}$ ) (7.9 and $6.5 \%$, respectively) and summed feature 3 (one or more of $\mathrm{C}_{16: 1} \omega 7 c, \mathrm{C}_{16: 1} \omega 6 c$ and iso- $\left.\mathrm{C}_{15: 0} 3-\mathrm{OH}\right)$, while these were not detected in strain BAV1 or FL2 (White et al., 2005). 10Methyl $\mathrm{C}_{16: 0}$ made up a large proportion of the cellular fatty acids in 'Dehalococcoides' sp. BAV1 and FL2 (21.3 and $24.4 \%$, respectively; White et al., 2005) but was not detected in strain BL-DC-8 or BL-DC- ${ }^{\mathrm{T}} . \mathrm{C}_{18: 1} \omega 9 c$ comprised a major fraction of the cellular fatty acids in strains BL-DC-8 and BL-DC- $9^{\mathrm{T}}$ (21.8 and $23.4 \%$, respectively) but was present in only trace amounts in 'Dehalococcoides' strains BAV1 and FL2 (0.4 and 0.5\%, respectively; White et al., 2005).

Additionally, strains BL-DC- 8 and BL-DC- ${ }^{\mathrm{T}}$ were shown to reductively dehalogenate polychlorinated alkanes (e.g. 1,2dichloropropane, 1,2-dichloroethane, 1,1,2-trichloroethane, 1,1,2,2-tetrachloroethane, 1,2,3-trichloropropane), but not monochlorinated alkanes (e.g. 1-chloropropane, 2-chloropropane), chlorinated alkenes (e.g. tetrachloroethene, trichloroethene, cis-dichloroethene, trans-dichloroethene, vinyl chloride) or chlorinated benzenes (1-chlorobenzene, 1,2-dichlorobenzene) (Yan et al., 2009). All dechlorination reactions determined to date appear to involve exclusively a dichlorelimination reaction mechanism (Yan et al., 2009). This range of compounds serving as electron acceptors and the presence of apparently exclusive dichlorelimination (involving simultaneous removal of two chlorines from adjacent carbon atoms and formation of a carbon-carbon double bond) for strains BL-DC-8 and BL-DC- $9^{\mathrm{T}}$ differs from the 'Dehalococcoides' strains reported to date, which can reductively dehalogenate chlorinated alkenes and chlorinated benzenes and employ a reaction mechanism involving sequential removal of one chlorine atom at a time (Maymó-Gatell et al., 1997, 1999, 2001; Adrian et al., 2000; He et al., 2003, 2005; Sung et al., 2006).

Based on these characteristics, strains BL-DC-8 and BLDC $-9^{T}$ are considered to represent a new genus distinct from the informally proposed genus 'Dehalococcoides'. It should be noted that any future valid publication of the name 'Dehalococcoides' would not be impacted by, nor would it impact, the genus Dehalogenimonas proposed herein.

Levels of 16S rRNA gene sequence similarity between strains BL-DC- 8 and BL-DC- $9^{\mathrm{T}}$ and members of recognized genera in the phylum Chloroflexi were extremely low, and phylogenetic distance readily served as a differentiating property (Fig. 1). Phenotypic characteristics also differentiated strains BL-DC-8 and BL-DC- $9^{\mathrm{T}}$ from recognized genera in the phylum Chloroflexi (Supplementary Table S1). Within the class Anaerolinea, members of the genera Anaerolinea (Sekiguchi et al., 2003; Yamada et al., 2006), Bellilinea (Yamada et al., 2007), Leptolinea (Yamada et al., 2006), Levilinea (Yamada et al., 2006) and Longilinea (Yamada et al., 2007) are filamentous, whereas cells of strains BL-DC-8 and BL-DC- $9^{\mathrm{T}}$ are irregular cocci. Additionally, members of the genera Anaerolinea and Bellilinea are thermophilic, while strains BL-DC-8 and BLDC- $-{ }^{\mathrm{T}}$ are mesophilic. At the time of writing, the class Caldilineae comprises a single genus with a single species, Caldilinea aerophila (Sekiguchi et al., 2003; Yamada et al., 2006), which can be differentiated from strains BL-DC-8 and BL-DC- $9^{\mathrm{T}}$ based on the fact that it is filamentous, thermophilic and facultatively aerobic (Supplementary Table S1).

Phenotypically, strains BL-DC-8 and BL-DC- $9^{\mathrm{T}}$ could be differentiated from the filamentous anoxygenic phototrophs of the genera Chloroflexus, Chloronema, Heliothrix, Oscillochloris and Roseiflexus on the basis of cell morphology (Supplementary Table S1). In addition, the type strains of species of the genera Chloroflexus, Heliothrix and Roseiflexus are thermophilic (Pierson \& Castenholz, 1974; Pierson et al., 1985; Hanada et al., 1995, 2002) whereas strains BL-DC-8 and BL-DC- $9^{\mathrm{T}}$ are mesophilic (Yan et al., 2009). Strains BL-DC-8 and BL-DC- $9^{\mathrm{T}}$ could be differentiated from the chemoheterotrophic genus Herpetosiphon on the basis that the latter has lower DNA G + C content and filamentous cell morphology and exhibits aerobic metabolism (Holt \& Lewin, 1968; Lewin, 1970; Holt \& Castenholz, 2001). 
Strains BL-DC-8 and BL-DC- $9^{\mathrm{T}}$ may be differentiated from members of the class Thermomicrobia on the basis that the species assigned to this class, namely Thermomicrobium roseum (Perry, 2001; Hugenholtz \& Stackebrandt, 2004) and Sphaerobacter thermophilus (Demharter et al., 1989; Hugenholtz \& Stackebrandt, 2004), are thermophilic, aerobic, rod-shaped and have appreciably higher DNA $\mathrm{G}+\mathrm{C}$ contents. In addition, Sphaerobacter thermophilus is Gram-positive (Demharter et al., 1989) whereas strains BLDC-8 and BL-DC-9 ${ }^{\mathrm{T}}$ stain Gram-negative.

On the basis of phylogenetic, chemotaxonomic and phenotypic data, strains BL-DC- 8 and BL-DC- $9^{\mathrm{T}}$ are clearly distinct from other genera in the phylum Chloroflexi. We suggest that strains BL-DC-8 and BL-DC$9^{\mathrm{T}}$ represent a novel species of a new genus, for which the name Dehalogenimonas lykanthroporepellens gen. nov., sp. nov. is proposed.

\section{Description of Dehalogenimonas gen. nov.}

Dehalogenimonas (De.ha.lo.ge.ni.mo'nas. L. prep. de away, off; N.L. n. halogenum halogen; L. fem. n. monas unit, monad; N.L. fem. n. Dehalogenimonas dehalogenating monad, reflecting the ability of these bacteria to dehalogenate chlorinated alkanes).

Cells are Gram-negative staining, non-motile, non-sporeforming, irregular cocci. Strictly anaerobic and mesophilic. Chemotrophic. Able to reductively dehalogenate chlorinated alkanes. Utilize $\mathrm{H}_{2}$ as an electron donor. The $\mathrm{G}+\mathrm{C}$ content of the genomic DNA is $53-54 \mathrm{~mol} \%$. On the basis of $16 \mathrm{~S}$ rRNA gene sequence comparisons, the genus belongs to the phylum Chloroflexi. The type species is Dehalogenimonas lykanthroporepellens.

\section{Description of Dehalogenimonas lykanthroporepellens sp. nov.}

Dehalogenimonas lykanthroporepellens (ly.kan.thro.po.re. pel'lens. Gr. n. lykanthropos werewolf; L. part. adj. repellens repelling; N.L. part. adj. lykanthroporepellens repelling werewolves, because compounds exhibiting a pungent garlic aroma are produced when these organisms grow in the presence of 1,2,3-trichloropropane as an electron acceptor and sulfide as a reducing agent, garlic being said to repel werewolves in some fiction literature).

Cells are $0.3-0.6 \mu \mathrm{m}$ in diameter. Able to reductively dehalogenate polychlorinated aliphatic alkanes, including 1,2,3-trichloropropane, 1,2-dichloropropane, 1,1,2,2-tetrachloroethane, 1,1,2-trichloroethane and 1,2-dichloroethane. When grown with 1,2,3-trichloropropane as an electron acceptor and sulfide as a reducing agent, the reactive intermediate allyl chloride is produced, which results in production of allyl alcohol, diallyl sulfide and diallyl disulfide. Does not utilize 1-chlorobenzene, 1chloropropane, 2-chloropropane, 1,2-dichlorobenzene, cis-1,2-dichloroethene, trans-1,2-dichloroethene, tetrachlorothene, trichloroethene or vinyl chloride as electron acceptors for growth. Growth is not supported by acetate, butyrate, citrate, ethanol, fructose, fumarate, glucose, lactate, lactose, malate, methanol, methyl ethyl ketone, propionate, pyruvate, succinate or yeast extract in the absence of $\mathrm{H}_{2}$. Resistant to ampicillin and vancomycin. The temperature range for growth is $20-34{ }^{\circ} \mathrm{C}$ (optimum 28 $34{ }^{\circ} \mathrm{C}$ ). The $\mathrm{pH}$ range for growth is $6.0-8.0$ (optimum $\mathrm{pH}$ 7.0-7.5). Growth occurs at $\mathrm{NaCl}$ concentrations up to at least $2 \%(\mathrm{w} / \mathrm{v})$. Major cellular fatty acids are $\mathrm{C}_{18: 1} \omega 9 c$, $\mathrm{C}_{16: 1} \omega 9 c, \mathrm{C}_{16: 0}$ and $\mathrm{C}_{14: 0}$. The $\mathrm{G}+\mathrm{C}$ content of the genomic DNA of the type strain is $54.0 \mathrm{~mol} \%$.

The type strain, BL-DC- $9^{\mathrm{T}}\left(=\right.$ ATCC BAA- $1523^{\mathrm{T}}=\mathrm{JCM}$ $15061^{\mathrm{T}}$ ), was isolated from chlorinated solvent-contaminated groundwater at the PetroProcessors of Louisiana, Inc. Superfund Site, located near Baton Rouge, LA, USA. BL-DC-8, isolated from the same source, is a second strain of the species.

\section{Acknowledgements}

This research was funded by the Governor's Biotechnology Initiative of the Louisiana Board of Regents grant BOR\#015 and NPC Services, Inc. We thank M. C. Henk and W. G. Henk for assistance with microscopy. We thank J. P. Euzéby for assistance with the etymology of the new names.

\section{References}

Adrian, L., Szewzyk, U., Wecke, J. \& Gorisch, H. (2000). Bacterial dehalorespiration with chlorinated benzenes. Nature 408, 580-583.

Bowman, K. S., Moe, W. M., Rash, B. A., Bae, H. S. \& Rainey, F. A. (2006). Bacterial diversity of an acidic Louisiana groundwater contaminated by dense nonaqueous-phase liquid containing chloroethanes and other solvents. FEMS Microbiol Ecol 58, 120-133.

Castenholz, R. W. (2001). Class I. “Chloroflexi”. In Bergey's Manual of Systematic Bacteriology, 2nd edn, vol. 1, p. 427. Edited by D. R. Boone, R. W. Castenholz \& G. M. Garrity. New York: Springer.

Demharter, W., Hensel, R., Smida, J. \& Stackebrandt, E. (1989). Sphaerobacter thermophilus gen. nov., sp. nov. A deeply rooting member of the actinomycetes subdivision isolated from thermophilically treated sewage sludge. Syst Appl Microbiol 11, 261-266.

Felsenstein, J. (1985). Confidence limits on phylogenies: an approach using the bootstrap. Evolution 39, 783-791.

Hanada, S., Hiraishi, A., Shimada, K. \& Matsuura, K. (1995). Chloroflexus aggregans sp. nov., a filamentous phototrophic bacterium which forms dense cell aggregates. Int J Syst Bacteriol 45, 676-681.

Hanada, S., Takaichi, S., Matsuura, K. \& Nakamura, K. (2002). Roseiflexus castenholzii gen. nov., sp. nov., a thermophilic, filamentous, photosynthetic bacterium that lacks chlorosomes. Int J Syst Evol Microbiol 52, 187-193.

He, J., Ritalahti, K. M., Yang, K.-L., Koenigsbeg, S. S. \& Löffler, F. E. (2003). Detoxification of vinyl chloride to ethene coupled to growth of an anaerobic bacterium. Nature 424, 62-64.

He, J., Sung, Y., Krajmalnik-Brown, R., Ritalahti, K. M. \& Löffler, F. E. (2005). Isolation and characterization of Dehalococcoides sp. strain FL2, a trichloroethene (TCE)- and 1,2-dichloroethene-respiring anaerobe. Environ Microbiol 7, 1442-1450.

Hendrickson, E. R., Payne, J. A., Young, R. M., Starr, M. G., Perry, M. P., Fahnestock, S., Ellis, D. E. \& Ebersole, R. C. (2002). Molecular 
analysis of Dehalococcoides $16 \mathrm{~S}$ ribosomal DNA from chloroethenecontaminated sites throughout North America and Europe. Appl Environ Microbiol 68, 485-495.

Holt, J. G. \& Castenholz, R. W. (2001). Genus I. Herpetosiphon Holt and Lewin 1968, 2408 ${ }^{\mathrm{AL}}$. In Bergey's Manual of Systematic Bacteriology, 2nd edn, vol. 1, pp. 445-446. Edited by D. R. Boone, R. W. Castenholz \& G. M. Garrity. New York: Springer.

Holt, J. G. \& Lewin, R. A. (1968). Herpetosiphon aurantiacus gen. et sp. n., a new filamentous gliding organism. J Bacteriol 95, 2407-2408.

Hugenholtz, P. \& Stackebrandt, E. (2004). Reclassification of Sphaerobacter thermophilus from the subclass Sphaerobacteridae in the phylum Actinobacteria to the class Thermomicrobia (emended description) in the phylum Chloroflexi (emended description). Int $J$ Syst Evol Microbiol 54, 2049-2051.

Jukes, T. H. \& Cantor, C. R. (1969). Evolution of protein molecules. In Mammalian Protein Metabolism, vol. 3, pp. 21-132. Edited by H. N. Munro. New York: Academic Press.

Kube, M., Beck, A., Zinder, S. H., Kuhl, H., Reinhardt, R. \& Adrian, L. (2005). Genome sequence of the chlorinated compound-respiring bacterium Dehalococcoides species strain CBDB1. Nat Biotechnol 23, 1269-1273.

Laakso, I., Seppänen-Laakso, T., Hiltunen, R., Müller, B., Jansen, H. \& Knobloch, K. (1989). Volatile garlic odor components: gas phases and adsorbed exhaled air analyzed by headspace gas chromatographymass spectrometry. Planta Med 55, 257-261.

Lapage, S. P., Sneath, P. H. A., Lessel, E. F., Skerman, V. B. D., Seeliger, H. P. R. \& Clark, W. A. (editors) (1992). International Code of Nomenclature of Bacteria (1990 Revision). Bacteriological Code. Washington, DC: American Society for Microbiology.

Lawson, L. D. (1996). The composition and chemistry of garlic cloves and processed garlic. In Garlic: The Science and Therapeutic Application of Allium sativum L. and Related Species, 2nd edn, pp. 37-107. Edited by H. P. Kock \& L. D. Lawson. Baltimore: Williams \& Wilkins.

Lewin, R. A. (1970). New Herpetosiphon species (Flexibacterales). Can J Microbiol 16, 517-520.

Ludwig, W., Strunk, O., Westram, R., Richter, L., Meier, H., Yadhukumar, Buchner, A., Lai, T., Steppi, S. \& other authors (2004). ARB: a software environment for sequence data. Nucleic Acids Res 32, 1363-1371.

Maymó-Gatell, X., Chien, Y., Gossett, J. M. \& Zinder, S. H. (1997). Isolation of a bacterium that reductively dechlorinates tetrachloroethene to ethene. Science 276, 1568-1571.

Maymó-Gatell, X., Anguish, T. \& Zinder, S. H. (1999). Reductive dechlorination of chlorinated ethenes and 1,2-dichloroethane by 'Dehalococcoides ethenogenes' 195. Appl Environ Microbiol 65, 3108-3113.

Maymó-Gatell, X., Nijenhuis, I. \& Zinder, S. H. (2001). Reductive dechlorination of cis-1,2-dichloroethene and vinyl chloride by 'Dehalococcoides ethenogenes'. Environ Sci Technol 35, 516-521.

Mesbah, M., Premachandran, U. \& Whitman, W. B. (1989). Precise measurement of the $\mathrm{G}+\mathrm{C}$ content of deoxyribonucleic acid by highperformance liquid chromatography. Int J Syst Bacteriol 39, 159-167.
Perry, J. J. (2001). Genus I. Thermomicrobium Jackson, Ramaley and Meinschein 1973, 34 ${ }^{\mathrm{AL}}$. In Bergey's Manual of Systematic Bacteriology, 2nd edn, vol. 1, pp. 448-450. Edited by D. R. Boone, R. W. Castenholz \& G. M. Garrity. New York: Springer.

Pierson, B. K. \& Castenholz, R. W. (1974). A phototrophic gliding filamentous bacterium of hot springs, Chloroflexus aurantiacus, gen. and sp. nov. Arch Microbiol 100, 5-24.

Pierson, B. K., Giovannoni, S. J., Stahl, D. A. \& Castenholz, R. W. (1985). Heliothrix oregonensis, gen. nov., sp. nov., a phototrophic filamentous gliding bacterium containing bacteriochlorophyll $a$. Arch Microbiol 142, 164-167.

Sasser, M. (1990). Identification of bacteria by gas chromatography of cellular fatty acids, MIDI Technical Note 101. Newark, DE: MIDI Inc.

Sekiguchi, Y., Yamada, T., Hanada, S., Ohashi, A., Harada, H. \& Kamagata, Y. (2003). Anaerolinea thermophila gen. nov., sp. nov. and Caldilinea aerophila gen. nov., sp. nov., novel filamentous thermophiles that represent a previously uncultured lineage of the domain Bacteria at the subphylum level. Int J Syst Evol Microbiol 53, 18431851.

Seshadri, R., Adrian, L., Fouts, D. E., Eisen, J. A., Phillippy, A. M., Methe, B. A., Ward, N. L., Nelson, W. C., Deboy, R. T. \& other authors (2005). Genome sequence of the PCE-dechlorinating bacterium Dehalococcoides ethenogenes. Science 307, 105-108.

Sung, Y., Ritalahti, K. M., Apkarian, R. P. \& Löffler, F. E. (2006). Quantitative PCR confirms purity of strain GT, a novel trichloroethene-to-ethene-respiring Dehalococcoides isolate. Appl Environ Microbiol 72, 1980-1987.

White, D. C., Geyer, R., Peacock, A. D., Hedrick, D. B., Koenigsberg, S. S., Sung, Y., He, J. \& Löffler, F. E. (2005). Phospholipid furan fatty acids and ubiquinone-8: lipid biomarkers that may protect Dehalococcoides strains from free radicals. Appl Environ Microbiol 71, 8426-8433.

Yamada, T., Sekiguchi, Y., Hanada, S., Imachi, H., Ohashi, A., Harada, H. \& Kamagata, Y. (2006). Anaerolinea thermolimosa sp. nov., Levilinea saccarolytica gen. nov., sp. nov. and Leptolinea tardivitalis gen. nov., sp. nov., novel filamentous anaerobes, and description of the new classes Anaerolineae classis nov. and Caldineae classis nov. in the bacterial phylum Chloroflexi. Int J Syst Evol Microbiol 56, 1331-1340.

Yamada, T., Imachi, H., Ohashi, A., Harada, H., Hanada, S., Kamagata, Y. \& Sekiguchi, Y. (2007). Bellilinea caldifistulae gen. nov., sp. nov. and Longilinea arvoryzae gen. nov., sp. nov., strictly anaerobic, filamentous bacteria of the phylum Chloroflexi isolated from methanogenic propionate-degrading consortia. Int J Syst Evol Microbiol 57, 2299-2306.

Yan, J., Rash, B. A., Rainey, F. A. \& Moe, W. M. (2009). Isolation of novel bacteria within the Chloroflexi capable of reductive dechlorination of 1,2,3-trichloropropane. Environ Microbiol 11, 833-843.

Zehnder, A. J. B. \& Wuhrmann, K. (1976). Titanium (III) citrate as a nontoxic oxidation-reduction buffering system for the culture of obligate anaerobes. Science 194, 1165-1166. 